\title{
Global Health Surveillance: Innovation and Coordination for Broad Health Impact
}

\author{
Ray L. Ransom¹, Olga L. Henao ${ }^{1}$, Leonard Peruski ${ }^{1}$, Ruth Kigozi ${ }^{2}$, David Blazes ${ }^{3}$, \\ William Bertrand ${ }^{4}$ and Joel Montgomery ${ }^{1}$
}

${ }^{1}$ Center for Global Health, Centers for Disease Control and Prevention, Atlanta, GA, USA; ${ }^{2}$ Infectious Disease Research Collaboration, Kampala, Uganda; ${ }^{3}$ Bill and Melinda Gates Foundation, Seattle, WA, USA; ${ }^{4}$ School of Public Health and Tropical Medicine, Tulane University, New Orleans, LA, USA

\section{Objective}

The session will discuss strategies for outbreak prevention, detection, and response for global health security and explore how these activities inform both domestic and international initiatives. Innovations in epidemiology, laboratory, informatics, investment, and coordination for disease surveillance will be discussed.

\section{Introduction}

Multiple agencies are involved in global disease surveillance and coordination of activities is essential to achieve broad public health impact. Multiple examples of effective and collaborative initiatives exist. The WHO/AFRO developed Integrated Disease Surveillance and Response (IDSR) framework, adopted by 43 of the 46 AFRO member states and applied in other WHO regions, was the first framework designed to strengthen national disease surveillance and response systems. The WHO International Health Regulations (IHR) 2005 are an agreement between 196 countries to prevent, detect and respond to the international spread of disease. In 2013 CDC worked with Uganda and Vietnam to demonstrate the development of surveillance, laboratory, and emergency response center capacity and link data systems for six outbreak prone diseases. More recently, the Global Health Security Agenda (GHSA) was launched with the support of 28 countries, WHO, OIE and FAO just as Ebola was beginning to emerge in West Africa. This panel brings together CDC, local implementing partners, academic technical partners, and international non-government donor to discuss current and evolving strategies for prevention, detection, and response activities needed for global health security.

\section{Keywords}

Surveillance; Informatics; Laboratory; Data Integration; Global

\section{*Ray L. Ransom}

E-mail: rransom@cdc.gov 